\title{
Aplicação de uma tecnologia educacional para a prevenção de acidentes causados por eixo de motor descoberto em um porto municipal de Belém-PA: Relato de experiência
}

\author{
Application of an educational technology for the prevention of motor shaft accidents \\ discovered in a municipal port of Belém-PA: Experience report
}

Aplicación de una tecnología educativa para la prevención de accidentes de eje motor descubiertos en un puerto municipal de Belém-PA: Informe de experiência

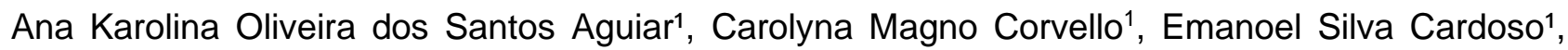
Keiliane Costa Oliveira ${ }^{1}$, Ana Alice Matias Ambé1, Bruno Jáy Mercês de Lima², Shirley Aviz de Miranda ${ }^{2}$, Barbara Cohen Monteiro ${ }^{1}$, Jessica de Nazaré Souza Ferreira', Eva Campos de Souza', Kamila Braga da Silva ${ }^{1}$, Malena Gabriele Duarte Duarte ${ }^{1}$, Nádia Oliveira Aires¹, Rosiany Pires França ${ }^{1}$, Yves de Luka Miranda dos Santos'1.

\section{RESUMO}

Objetivo: Relatar a experiência de acadêmicos de enfermagem na criação e aplicação de uma tecnologia educacional para a prevenção de acidentes causados por eixo de motor descoberto em transportes fluviais. Relato de experiência: Inicialmente foram identificados os principais determinantes que envolvem o acidente com o eixo de motor descoberto, o escalpelamento, e a partir de então, pensado no desenvolvimento de uma ação educativa com o uso de uma tecnologia que levasse de forma clara e eficiente informações de promoção e prevenção de saúde. A segunda etapa foi à aplicação da ação educativa nos transportes fluviais no Porto Municipal de Icoaraci - PA que utilizou de duas tecnologias: a tecnologia leve, que foi uma sala de espera flutuante e tecnologia leve-dura em forma de cartazes e adesivos colados nas embarcações. Considerações finais: Evidenciou-se que é escolha da tecnologia educacional aplicada proporcionou um melhor aprendizado ao publico alvo. Destaca-se também a compreensão da necessidade de mais ações educativas para combater o escalpelamento, que infelizmente é um acidente ainda muito comum na região amazônica.

Palavras-chave: Couro cabeludo, Promoção da saúde, Tecnologia educacional.

\begin{abstract}
Objective: To report the experience of nursing academics in the creation and application of an educational technology for the prevention of uncovered motor shaft accidents in river transport. Experiencereport: Initially, the main determinants involving the uncovered motor shaft accident, scalping, were identified, and from then on, thought was given to developing an educational action using a technology that would lead clearly and efficiently health promotion and prevention information. The second stage was the application of the educational action on river transport in the Icoaraci Municipal Port - PA that used two technologies: light technology, which was a floating waiting room and light-hard technology in the form of posters and stickers glued to the boats. Final considerations: It was evidenced that the choice of applied educational technology provided a better learning for the target audience. Also noteworthy is the understanding of the need for more educational actions to combat scalping, which unfortunately is still a very common accident in the Amazon region.
\end{abstract}

Keywords: Scalp, Health promotion, Educational technology.

${ }^{1}$ Centro Universitário Metropolitano da Amazônia (UNIFAMAZ). Belém-Pará, Brasil.

*E-mail: akarolinaoliveira1908@gmail.com

2 Universidade Estadual do Pará (UEPA). Belém-Pará, Brasil. 


\section{RESUMEN}

Objetivo: Informar sobre la experiencia de los académicos de enfermería en la creación y aplicación de una tecnología educativa para la prevención de accidentes de motor descubierto en el transporte fluvial. Informe de experiencia: Inicialmente, se identificaron los principales determinantes que involucran el accidente descubierto del eje del motor, el scalping, y desde entonces, se pensó en desarrollar una acción educativa utilizando una tecnología que conduzca de manera clara y eficiente. información sobre promoción y prevención de la salud. La segunda etapa fue la aplicación de la acción educativa sobre el transporte fluvial en el Puerto Municipal de Icoaraci - PA que utilizó dos tecnologías: la tecnología de la luz, que era una sala de espera flotante y la tecnología resistente a la luz en forma de carteles y pegatinas pegados a los barcos. Consideraciones finales: se evidenció que la elección de la tecnología educativa aplicada proporcionó un mejor aprendizaje para el público objetivo. También es digno de mención la comprensión de la necesidad de más acciones educativas para combatir el cuero cabelludo, que desafortunadamente sigue siendo un accidente muy común en la región amazónica.

Palabras clave: Cuero cabelludo, Promoción de la salud, Tecnología educativa.

\section{INTRODUÇÃO}

A região amazônica é constituída pelo rio Amazonas e seus principais afluentes onde há rios, igarapés e furos, distribuídos por toda a região norte e região centro-oeste do país sendo a principal via de transporte de populações ribeirinhas. Denominam-se como ribeirinhos as comunidades que residem às margens dos rios. A principal fonte de renda dessa população é a extração e venda de produtos naturais, cultivados frequentemente em seu próprio quintal, e para a comercialização de tais produtos, eles utilizam de pequenas embarcações como meio de transporte e para que possam exercer suas atividades de trabalho e tarefas diárias (FERREIRA SC, et al. 2014).

Habitualmente, as famílias ribeirinhas confeccionam o seu próprio barco e, culturalmente as instruções do manejo do veículo é passado a cada geração. Portanto, crianças e mulheres não fazem viagem apenas como passageiras, mas também como aprendiz, auxiliando o piloto. Uma das funções frequentes é a retirada da água acumulada próxima ao motor do barco, feita com algum pequeno recipiente, dessa forma, seus cabelos ficam expostos a parte descoberta do motor, levando ao risco do acidente mais recorrente, que é o escalpelamento. $O$ escalpelamento é um acidente em que o escalpo humano é retirado de forma brusca, na maior parte dos casos atinge mulheres e crianças ribeirinhas que possuem cabelos longos. Nas embarcações, isso ocorre quando o eixo do motor não está adequadamente protegido, sugando e arrancando parte ou todo o couro cabeludo da vítima, podendo levar a deformação da face, atingindo as sobrancelhas, orelhas e há casos de hemorragias profundas, podendo resultar em óbito (SILVA SP e BARROSO IC, 2013).

O índice de escalpelamento na infância é alto, em um estudo observacional transversal, retrospectivo, descritivo, incluindo 62 pacientes vítimas de escalpelamento evidencia que, 56,4\% das pacientes vítimas do acidente tinham idade entre 7 à 16 anos. Ou seja, grande parte dos acidentes acontece com mulheres, mais precisamente crianças, que tem não apenas a infância comprometida, como todo o resto de suas vidas. (CUNHA CB, 2012; MAGNO LDP, 2012).

As consequências do escalpelamento são as mais diversas possíveis e se estendem por todo o contexto biopsicossocial. As vítimas do acidente passam a enfrentar sequelas físicas, psíquicas e sociais, não só durante o tratamento, como no decorrer de suas vidas, pois acarreta danos significativos à autoestima, à identidade pessoal, à nova percepção corporal, a aceitação, ao humor, à interação social eaté mesmo às relações afetivas. Em vista disso, compreende-se a importância de trabalhar na prevenção e cuidados a serem adotados para que o acidente seja evitado (FERREIRA SC, et al., 2014; SILVA CG, 2014).

Os primeiros casos de acidentes por escalpelamento foram registrados na década de 1960, quando os barcos à vela foram substituídos por embarcações a motor. Porém somente a partir de 1990, com o trabalho da Organização da Sociedade Civil de Interesse Público (OSCIP) Sarapó, que o assunto ganhou visibilidade na mídia. A OSCIP, por meio de mobilizações, campanhas de prevenção e no amparo ao tratamento das vítimas, chamou a atenção do Poder Público, dos próprios ribeirinhos e, inclusive, da Marinha do Brasil, para 
a gravidade do fato. Nas campanhas profiláticas ocorre a distribuição de material que visa orientar a população ribeirinha, por meio de cartazes, folders, panfletos, entre outros, que são distribuídos em escolas, igrejas, hospitais e para a comunidade em geral (MORAES LC, 2015).

O Governo Federal, através da Lei oㅜ 11.970, de 6 de julho de 2009, estabeleceu a obrigatoriedade da instalação de proteções em torno de áreas móveis e do eixo do motor das embarcações que trafegam pelos rios. Em 2010, o Centro de Instrução Almirante Braz de Aguiar (CIABA), lançou a Campanha Nacional de Combate ao Escalpelamento, que tinha como objetivo disseminar informações fixando peças publicitárias em barcos e pontos de embarque/desembarque de passageiros. Para isso a Marinha realizou mutirões em parceria com diversos órgãos governamentais para a distribuição e instalação gratuita de dispositivos com cobertura metálica para acoplar no eixo do motor, nas regiões de ocorrência dos acidentes. Entretanto algumas vezes, os acidentes sequer são informados aos órgãos estaduais responsáveis pela prevenção e manutenção da saúde pública, ficando restritos ao próprio município (GUIMARÃES AGM, 2012; OLIVEIRA LS, 2016).

Faz-se necessário que no âmbito acadêmico, principalmente na área da enfermagem, haja mais estudos acerca do assunto, pois há uma escassez de informações a respeito de todos os aspectos que envolvem essas vítimas, nem mesmo nos Descritores em Ciências da Saúde (DECS), foi possível encontrar mais sobre o tema escalpelamento, sendo uma questão de saúde pública e pouco explorada, é um assunto que merece mais atenção, divulgação, políticas públicas eficazes para obtenção de medidas profiláticas eficientes, que reduzam esses acidentes que infelizmente, são comuns em nossa região amazônica (MARTISN MM e SILVA MA, 2013).

O objetivo deste trabalho é descrever a experiência de acadêmicos do quinto período do curso de enfermagem, no desenvolvimento e aplicação de uma tecnologia educacional através de uma ação educativa em saúde acerca de medidas de prevenção a acidentes causados por eixo de motor descoberto, escalpelamento em um porto de Belém do Pará.

\section{RELATO DE EXPERIÊNCIA}

Neste relato de experiência foi identificado que para efetividade do trabalho de prevenção dos acidentes, as comunidades locais deveriam articular com os representantes das secretarias municipais de Educação e Saúde, líderes comunitários, associações de pescadores e todas as instituições que estejam e possam estar envolvidas na causa, pois as ações devem ser pensadas junto aos sujeitos envolvidos na realidade.

Contando inclusive com a participação e apoio das igrejas (evangélica e católica) para que reforcem em seus discursos o cuidado com o uso de cabelos longos, que se entende na religião como, véu. Os representantes religiosos dessas comunidades têm forte influência no que concerne a vida no geral dos membros de suas igrejas, logo podem agir como multiplicadores das informações relevantes à prevenção dos acidentes (ALMEIDA EM, 2016).

Desse modo foi pensado em uma ação educativa com uso de uma tecnologia com informações para orientar as pessoas sobre medidas preventivas. Para isso, criamos uma tecnologia educacional com a proposta de contribuir no combate a acidentes por eixo de motor descoberto, o escalpelamento. As tecnologias educacionais são fundamentais no trabalho do enfermeiro e podem ser classificadas como leve, leve-dura e dura. As tecnologias duras referem-se aos instrumentos e equipamentos que serão utilizados. As leve-duras são as aplicações dos conhecimentos e saberes e o modo como cada profissional utilizam esses conhecimentos. As tecnologias leves correspondem às relações entre os indivíduos, as quais são construídas durante a produção do cuidado (RITTER CB, et al., 2014).

A segunda etapa foi à realização da ação educacional nos transportes fluviais do Porto Municipal de Icoaraci - PA, que aconteceu em uma sexta-feira, pois concluímos que de segunda a sexta o fluxo maior de pessoas no porto municipal, são de moradores das comunidades ribeirinhas, sendo eles nosso público alvo, pois podem está convivendo em situações de risco diariamente devido suas rotinas, e no horário com o maior fluxo de passageiros e embarcações no porto.Primeiramente utilizamos uma tecnologia leve, que foi a sala 
de espera flutuante, que ocorreu dentro das embarcações, com os passageiros que estavam aguardando a saída do barco, e falamos sobre escalpelamento, visando sensibilizá-los quanto aos riscos que levam ao acidente e orientá-los quanto às medidas de prevenção que devem ser tomadas durante as viagens. $E$ confeccionamos uma tecnologia leve-dura em forma de cartazes, para as embarcações grandes e com grande fluxo de passageiros e adesivos para as de pequeno porte, ambos foram colados nas embarcações.

Entramos em 40 embarcações no total, e nem todas tinham finalidade única de transporte de passageiros, algumas apenas transporte de carga, mas que ainda assim, tendo uma quantidade de pessoas circulantes no transporte. Abordamos de maneira cordial, pois não queríamos causar desconforto e nem parecermos fiscais, pedíamos para conversar com os encarregados dos barcos e passageiros onde apresentávamos o conteúdo das tecnologias, após pedíamos autorização para colarmos os cartazes/adesivos. Duas dessas embarcações não estavam com o eixo do motor coberto, tentamos conversar com seus donos. Ouviram-nos, ainda que visivelmente impacientes e não permitiram que colocássemos o adesivo, disseram que os mesmos fariam a colagem, assim levaram os adesivos, infelizmente não temos registro se de fato houve a colagem no barco, assim como fizemos nos demais, pois os mesmos logo partiram do porto

A associação dos barqueiros do trapiche municipal também permitiu a colagem nos pontos de venda de passagem, trazendo visibilidade das informações para as pessoas precisam se direcionar para compra do passaporte para os barcos. Fomos parados no corredor do porto por uma diretora de uma escola ribeirinha que nos pediu cartazes e adesivos para levar para escola e realizar um trabalho com as crianças e pais, ou seja, a tecnologia foi mais longe que nós mesmos.

\section{DISCUSSÃO}

O uso das tecnologias educacionais cresceu durante os últimos anos, principalmente em meio a enfermagem trazendo o enfermeiro como educador que utiliza desse meio tecnológico diversas formas de fundamentar estudos e práticas de saúde de maneira mais fácil visando promover uma melhora do cuidado em saúde. As tecnologias possibilitam que o enfermeiro entenda e atenda as necessidades das pessoas, quando usadas adequadamente. Para tanto, seu desenvolvimento, utilização e evolução têm como foco a junção entre o desenvolvimento humano e o tecnológico. Logo, entende-se que as tecnologias são uma ferramenta para auxiliar e facilitar a relação entre os seres humanos, sem afetar as relações interpessoais (ÁFIO ACE, et al., 2014).

A escolha das tecnologias do tipo sala de espera e cartazes/adesivos utilizadas na ação educativa salienta sobre a importância de produção de tecnologias educacionais que direcionem a qualidade do cuidado, pois contribuem diretamente para a eficácia, efetividade e segurança do cuidado, ou seja, utilizadas de maneira adequada criam condições que contribuem para o atendimento adequado e eficaz do que é transmitido através delas. Por isso, os pontos das colagens foram estratégicos para que pudessem chamar a atenção da população que trafega pelo porto e utiliza das embarcações para que as mesmas detenham dos cuidados adequados e possam transmiti-los para as demais pessoas, levando clareza e seguras das informações (SILVA DML, et al., 2017).

É importante ressaltar o fato de termos encontrados duas embarcações, aparentemente disponíveis para navegação sem a cobertura correta do eixo do motor, nos traz um questionamento quanto à de fiscalização que deveria ser realizada pelos rios. A Marinha do Brasil é a organização militar que detém da Autoridade Marítima cabendo-Ihe executar ações de Inspeção Naval objetivando a fiscalização do cumprimento daLei no 9.537, de 11 de dezembro de 1997 que se refere a segurança do tráfego aquaviário e a salvaguarda da vida humana. Oliveira LS (2016) aponta que há um temor desses proprietários de terem seu meio de transporte e fonte de renda apreendidos por irregularidades por isso evitam levar suas embarcações para que seja instalada a cobertura disponibilizada pela marinha.

Com isso, destaca-se que as ações devem acontecer no interior, na zona rural, nas vilas e comunidades ribeirinhas, pois esse é o público alvo que devemos atingir. Realizar simpósios, colóquios, encontros, seminários, mesas redondas dentro dessas comunidades, para que o público participante sejam os 
ribeirinhos, que são os maiores interessados nos assuntos, pois nem sempre se pode discutir de maneira fidedigna tais problemas se não formos afundo, e que inovador e transformador seria realizar atividades como essas pelos nossos rios da Amazônia paraense objetivando evitar e reduzir os riscos ao acidente (ALMEIDA EN, 2016).

A promoção das campanhas de prevenção contra o acidente deve contar com pequenas orientações como: prender o cabelo em forma de coque, não se aproximar do eixo do motor, cobrar do barqueiro a cobertura do eixo do motor, e fiscalização do poder público; orientação em como agir em casos de escalpelamento, não colocar remédio no local lesionado, cobrir a lesão com um pano limpo e levar a vítima a unidade de saúde ou hospital mais próximo (OLIVEIRA LS, 2016).

A aplicação dessas tecnologias visa tornar os ouvintes/leitores, multiplicadores das informações repassadas, para que o número de acidentes possa diminuir nos nossos rios amazônicos, pois há impactos sociais, físicos e psicológicos que ele pode causar, haja vista que o escalpelamento é um acidente que deixa sequelas irreversíveis na vida da vitima.Partilhamos com nossos ouvintes que os cuidados em a saúde mental das vítimas são de suma importância, uma vez que trata-se de um processo que requer cuidado, paciência, compreensão, tentativa de amenizar a dor da vítima, pois esse processo é lento e doloroso tanto fisicamente quanto mentalmente, além de afetar a vida social, pois muitas sofrem com bullying, adquirindo uma baixa autoestima ,necessitando de acompanhamento psicológico para evitar possíveis patologias que possam se manifestar devido ao transtorno causado pelo acidente (RIBEIRO NS, 2009; SILVA CG, 2014).

A experiência obtida nessa ação educativa em saúde sobre medidas preventivas para evitar acidentes causados por eixo de motor descoberto, em um porto municipal de Belém do Pará, não poderia ser mais significativa para nós quanto acadêmicos, gostaríamos de poder mensurar até onde a informação chegará, por quanto tempo os cartazes e adesivos ficaram nas embarcações, quantos pessoas irão parar e ler, mas infelizmente são questionamentos que ficaram sem uma resposta exata nesse momento.

\section{CONSIDERAÇÕES FINAIS}

Conclui-se que é escolha da tecnologia educacional aplicada proporcionou um melhor aprendizado ao publico alvo, sendo satisfatória não somente para eles, mas também no que concerne a nós futuros enfermeiros, desenvolvendo o papel da enfermagem quanto à promoção e prevenção em saúde, não se restringindo somente ao âmbito ambulatorial ou hospitalar. Destaca-se a compreensão da necessidade de mais ações educativas para combater o escalpelamento, que infelizmente é um acidente ainda muito comum na região amazônica.

\section{REFERÊNCIAS}

1. ÁFIO ALC, et al. Análise do conceito de tecnologia educacional em enfermagem aplicada ao paciente. Northeast Network Nursing Journal. 2014, vol.15.

2. ALMEIDA EN. O Corpo Escalpelado: possibilidades e desafios da ação docente com meninas vítimas de escalpelamento na Amazônia paraense ribeirinha. [Dissertação]. Belém-PA: Universidade Federal do Pará, 2016.

3. CUNHA CB. Perfil epidemiológico de pacientes vítimas de escalpelamento tratados na Fundação Santa Casa de Misericórdia do Pará. Rev. Bras. Cir. Plást. São Paulo, 2012; 27(1).

4. FERREIRA CS, et al. Saúde mental das vítimas de escalpelamento por eixo de motor de barco na região amazônica, 2014.

5. GUIMARÃES AGM. Mulheres da Amazônia: o drama do escalpelamento. Fortaleza: EDUCERE; EDMETA, 2012.

6. MAGNO LDP, et al. Escalpelamento nos rios da Amazônia: um problema de saúde pública. Revista paraense de medicina, Belém. 2012.

7. MARTINS MM, SILVA MA, Possibilidades de atuação da enfermagem em situações de escalpelamento. Estudos, Goiânia. 2013; 40(4): 341-365.

8. MORAES LC, et al. Meninas escalpeladas nos rios da Amazônia Paraense: o papel da mídia na prevenção de acidentes. Centro Internacional de Semiótica e Comunicação - CISECO IV Colóquio Semiótica Das Mídias. ISSN 2317-9147, Japaratinga - Alagoas. 2015. 
9. OLIVEIRA LS. Escalpelamento: Política Pública Para A População. R. Defensoria Públ. União Brasília. 2016 ; 9 : 1 504.

10. Ribeiro NS. Necessidade e dilemas das famílias vítimas de escalpelamento atendidas na FSCMP: desafios para o serviço social. Monografia (Trabalho de conclusão de curso) - Universidade Federal do Pará, Belém, 2009.

11. SILVA CG, et al. O escalpelamento e suas repercussões: uma análise sobre a identidade das vítimas e as contribuições da terapia ocupacional. Anais do III Congresso de Educação em Saúde da Amazônia (COESA), Universidade Federal do Pará, Belém, 2014. ISSN 2359-084X. 2012.

12. SILVA, DML et al. Tecnologias educacionais na assistência de enfermagem em educação em saúde: revisão integrativa. Ver. Enferm. UFPE ONLINE. 2017; 11, supl.2: 1044-1051.

13. SILVA SP, BARROSO IC. O padrão estético como fator estigmatizante de mulheres vítimas de escalpelamento em áreas ribeirinhas de Macapá e Santana, 2013; 6: 89-101. 